\title{
Cationic ionene as n-dopant agent of
}

\section{poly (3,4-ethylenedioxythiophene)}

\section{Maricruz G. Saborío, ${ }^{1,2}$ Oscar Bertran, ${ }^{3}$ Sonia Lanzalaco, ${ }^{1,2}$}

\section{Marleen Häring, ${ }^{4}$ David Díaz Díaz, ${ }^{4,5, *}$ Francesc Estrany, ${ }^{1,2, *}$ and Carlos Alemán ${ }^{1,2, *}$}

${ }^{1}$ Departament d'Enginyeria Química, EEBE, Universitat Politècnica de Catalunya, C/ Eduard Maristany 10-14, Ed. I2, 08019 Barcelona, Spain

${ }^{2}$ Barcelona Research Center for Multiscale Science and Engineering, Universitat Politècnica de Catalunya, Eduard Maristany 10-14, 08019 Barcelona, Spain

${ }^{3}$ Departament de Física, EEI, Universitat Politècnica de Catalunya, Av. Pla de la Massa, 8, 08700 Igualada, Barcelona, Spain

${ }^{4}$ Institut für Organische Chemie, Fakultät für Chemie und Pharmazie, Universität Regensburg, Universitätsstr. 31, 93053 Regensburg, Germany

${ }^{5}$ IQAC-CSIC, Jordi Girona 18-26, 08034 Barcelona, Spain

*Corresponding authors: david.diaz@chemie.uni-regensburg.de francesc.estrany@upc.edu and carlos.aleman@upc.edu 


\begin{abstract}
We report the reduction of poly(3,4-ethylenedioxythiophene) (PEDOT) films with a cationic 1,4-diazabicyclo[2.2.2] octane-based ionene bearing $\quad N, N^{\prime}$-(metaphenylene)dibenzamide linkages ( $m \mathrm{PI}$ ). Our main goal is to obtain n-doped PEDOT using a polymeric dopant agent rather than small conventional tetramethylammonium (TMA), as is usual. This has been achieved using a three-step process, which has been individually optimized: (1) preparation of p-doped (oxidized) PEDOT at a constant potential of $+1.40 \mathrm{~V}$ in acetonitrile with $\mathrm{LiClO}_{4}$ as electrolyte; (2) dedoping of oxidized PEDOT using a fixed potential of $-1.30 \mathrm{~V}$ in water; and (3) redoping of dedoped PEDOT applying a reduction potential of $-1.10 \mathrm{~V}$ in water with $m$ PI. The resulting films display the globular appearance typically observed for PEDOT, $m$ PI being structured in separated phases forming nanospheres or ultrathin sheets. This organization, which has been supported by atomistic Molecular Dynamics simulations, resembles the nanosegregated phase distribution observed for PEDOT $\mathrm{p}$-doped with poly(styrenesulfonate). Furthermore, the doping level achieved using $m$ PI as doping agent is comparable to that reached with TMA, even though the ionene provides distinctive properties to the conducting polymer. For example, films redoped with $m \mathrm{PI}$ exhibit much more hydrophilicity than the oxidized ones, whereas films redoped with TMA are hydrophobic. Similarly, films redoped $m$ PI exhibit the highest thermal stability, while those redoped with TMA show a thermal stability that is intermediate between the latter and dedoped PEDOT. Overall, the incorporation of $m$ PI polycation as n-dopant into PEDOT has important advantages for modulating the properties of this emblematic conducting polymer.
\end{abstract}




\section{INTRODUCTION}

Among conjugated polymers (CPs), doped poly(3,4-ethylenedioxythiophene) (Scheme 1), abbreviated PEDOT, is one of the most studied because of its great environmental stability, electrical conductivity, electrochemical activity, thermoelectric behavior and high specific capacitance. ${ }^{1-5}$ Additionally, PEDOT offers two fundamental advantages as compared to unsubstituted polythiophene (PTh) or other PTh derivatives: (1) the fused dioxane ring blocks the $\beta$-position of the thiophene ring and prevents from the formation of $\alpha-\beta$ linkages during the polymerization, which makes the resulting polymer well-defined from a regiochemical point of view; and (2) the fused dioxane ring and the electron-donating effects provided by the oxygen atoms cause the selfrigidification of polymer chains, resulting in a gain of aromaticity and in a reduction of the oxidative doping potential (p-doping) ${ }^{6}$

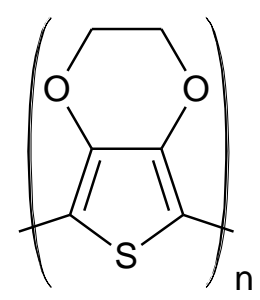

Scheme 1: Chemical structure of PEDOT

Oxidized PEDOT (p-doped) has been prepared using small anions as doping agents (e.g. $\mathrm{ClO}_{4}^{-}, \mathrm{NO}_{3}^{-}, \mathrm{SO}_{4}^{2-}$ and citrate), electrochemical activity being dominated by ionic and solvent movements. ${ }^{7-9}$ The oxidation towards the $\mathrm{p}$-doped state causes the access of solvated dopant anions inside the polymeric matrix, while they scape upon reduction towards the undoped (neutral) state. Similarly, large and even macromolecular dopant anions, as for example dodecyl sulfate and poly(styrenesulfonate) (PSS), can be successfully used to produce p-doped PEDOT. ${ }^{10-12}$ The PEDOT:PSS complex consists of a phase segregated structure in which $\sim 30 \mathrm{~nm}$ diameter conductive PEDOT-rich 
polycationic domains are encapsulated by $\sim 1 \mathrm{~nm}$ thick PSS-rich polyanionic shells. ${ }^{13}$ The domains are embedded in an electronically insulating PSS matrix loosely crosslinked by hydrogen bonding. ${ }^{14}$ PSS-doped PEDOT polymer mixtures exhibit good film forming properties, moderate to high conductivity, high visible light transmittance and excellent stability and, therefore, are suitable for use as low-cost wearable sensors, ${ }^{15}$ electrodes, ${ }^{16,17}$ soft actuators, ${ }^{19,20}$ and as hole injection/extraction material in organic optoelectronics. $^{21}$

PEDOT is one the few CPs that are both p- and n-dopable. However, although reduced PEDOT (n-doped) was reported more than two decades ago by Inganäs and coworkers, ${ }^{22}$ the number of studies on this material is very scarce. ${ }^{22-27}$ More specifically, these studies were focussed on the spectroscopic, electrical and electrochemical properties of negatively charged PEDOT prepared using small tetra-alkylammonium cations as electrolyte. ${ }^{22-26}$ However, experiments using other CPs revealed that the role of the electrolytic agent and the solvent in the n-doping is even higher than in the pdoping. ${ }^{28-30}$ According to these observations, in a recent study, Suárez-Herrera and coworkers ${ }^{27}$ showed that the negative charge of the n-doped PEDOT stabilizes when imidazolium-containing ionic liquids are used as dopant cations, resulting in a high ndoping concentration with respect to than obtained with tetra-alkylammonium cations. $^{22-26}$

On the other hand, ionenes are high charged polymers (polyelectrolytes) in which the ionic groups form part of the macromolecular backbone. ${ }^{31}$ More specifically, the term ionene frequently invokes to polycations having quaternary ammonium in the backbone, which have been applied in diverse applications (e.g. antibacterial agents and functional gels). ${ }^{31,32}$ The properties of ionenes are determined, among others, by the charge distribution, the molecular weight, the hydrogen bonding capability and the type of 


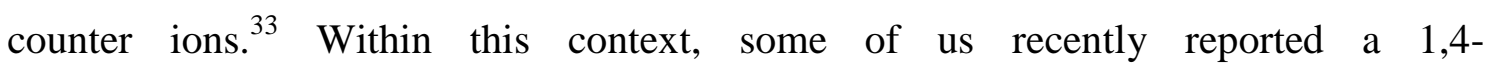
diazabicyclo[2.2.2]octane (DABCO)-based ionene bearing $\quad N, N^{\prime}$-(metaphenylene)dibenzamide linkages, hereafter denoted $m \mathrm{PI}$ (Scheme 2). ${ }^{34,35} \mathrm{We}$ found that, although $m$ PI chains organize forming regions dominated by ionene $\cdots$ ionene intermolecular interactions, these domains are much less frequent than in the isomer with ortho topology in the $N, N^{\prime}$-(phenylene)dibenzamide linkages. This particular characteristic suggests that $m \mathrm{PI}$ polycation is suitable to interact favorably with other macromolecular compounds forming stable mixtures, whereas chains of the ortho isomer were found to aggregate rapidly forming gels with good properties. ${ }^{34,35}$ Furthermore, $m \mathrm{PI}$ bears two close positive charges per repeat unit located at quaternary ammonium moieties (Scheme 2), making this polycation a very suitable candidate for the generation of negative charges at the PEDOT chains.<smiles>CCc1ccc(C(=O)Nc2cccc(NC(=O)c3ccc(C[N+]45CC[N+](C)(CC4)CC5)cc3)c2)cc1</smiles>

Scheme 2: Chemical structure of $m \mathrm{PI}$

In this study we propose the preparation of n-doped PEDOT using $m \mathrm{PI}$ as a macromolecular dopant agent for its incorporation into pristine oxidized PEDOT films. After optimizing the preparation process to achieve the highest n-doping concentration, the properties of the resulting material are compared with those of n-doped PEDOT obtained using conventional tetramethylammonium (TMA). Results allow us to conclude that the incorporation of $m$ PI polycations into PEDOT have important advantages, as for example the improvement of the thermal stability and the preparation of superhydrophilic electroactive surfaces. 


\section{AQUI RESULTS AND DISCUSSION}

\section{Preparation of p-doped PEDOT and $m P I$}

Oxidized PEDOT films were prepared by applying an optimized procedure reported in the literature: ${ }^{36}$ potentiostatic electropolymerization at $+1.40 \mathrm{~V}$ in an acetonitrile solution containing $10 \mathrm{mM}$ of monomer and $100 \mathrm{mM} \mathrm{LiClO}_{4}$. The polymerization charge was adjusted to $0.96 \mathrm{C}\left(480 \mathrm{mC} / \mathrm{cm}^{2}\right)$. The polymer doped with $\mathrm{ClO}_{4}^{-}$presents a film thickness of $6.8 \pm 0.6 \mu \mathrm{m}$. The monomeric units support average positive charges of +0.16 , balanced with $\mathrm{ClO}_{4}^{-}$counterions (i.e. 6.25 monomeric units per $\mathrm{ClO}_{4}^{-}$anion). Details on the complete electrochemical setup are provided in the Supporting Information.

A complete description of the procedure for the preparation of $m \mathrm{PI}$ was detailed in a previous work. ${ }^{34}$ The molecular weights of the resulting $m \mathrm{PI}$, which were calibrated using poly(methyl methacrylate) standards are: $\mathrm{M}_{\mathrm{n}}=5.0 \cdot 10^{3} \mathrm{Da}, \mathrm{M}_{\mathrm{w}}=1.2 \cdot 10^{3} \mathrm{Da}$ and the average number of chemical repeating units per chain $(n$ in Scheme 2$)=7$.

\section{Dedoping of p-doped PEDOT films}

Choronopotentiograms of the electropolymerized p-doped PEDOT were recorded in water with $10 \mathrm{mM} \mathrm{LiClO}_{4}$ at $-1.0 \mathrm{~mA}$ to characterize the dedoping process. The change in the slope of the resulting potential-time profiles indicated that the dedoping of $\mathrm{p}$ doped PEDOT occurs after $\sim 135 \mathrm{~s}$ at a potential close to $-0.8 \mathrm{~V}$ (Figure 1a). Accordingly, the dedoping occurs at a slow rate allowing the polymer chains to rearrange and minimize the film stress. Besides, Figure 1a indicates that the reduction potential in water with $10 \mathrm{mM} \mathrm{LiClO}_{4}$ stabilizes at around $-1.40 \mathrm{~V}$ after $\sim 150 \mathrm{~s}$. The charge consumed during the dedoping, $Q_{d d o p}$, in water with $10 \mathrm{mM} \mathrm{LiClO}_{4}$ applying $1.0 \mathrm{~mA}$ during $150 \mathrm{~s}$, once the reduction potential reached the value of $-1.40 \mathrm{~V}$, was - 
$0.132 \mathrm{C}$ (Figure 1b). Application of a second chronopotentiometric dedoping using the same experimental conditions, intensity and time, led to a reduction potential of $-0.68 \mathrm{~V}$ only (Figure 1b), indicating that such additional process does not represent a significant improvement.

Chronopotentiometric results (Figures 1a-b, were used to propose the experimental conditions used for new chronoamperometric dedoping assays. Specifically, these measures were performed fixing the reduction potential at $-1.10,-1.20,-1.30,-1.40,-$ 1.50 or $-1.60 \mathrm{~V}$ during $100 \mathrm{~s}$ in a $10 \mathrm{mM} \mathrm{LiClO}_{4}$ water solution. Figure 1c represents the value of $Q_{d d o p}$ obtained at each of such potentials. As it can be seen, the chronoamperometric dedoping is considerably more pronounced than the chronopotentiometric one, independently of the reduction potential (i.e. $Q_{d d o p}<-0.132$ $\mathrm{C}$ for all reduction potentials). In spite of this, the reduction potential has a very remarkable effect in chronoamperometric $Q_{d d o p}$ values, which range from $-0.192 \mathrm{C}$ for $1.60 \mathrm{~V}$ to $-0.264 \mathrm{C}$ for $-1.30 \mathrm{~V}$.

According to results displayed in Figure 1, the application of a fixed potential of $1.30 \mathrm{~V}$ during $100 \mathrm{~s}$ in a $10 \mathrm{mM} \mathrm{LiClO}_{4}$ water solution was selected as the most efficient procedure for the dedoping of p-doped PEDOT. Hereafter, this dedoping procedure is the only applied for subsequent studies.

The surface morphology of p-doped (as prepared) and dedoped PEDOT samples is displayed in Figure $2 \mathrm{a}$ and $2 \mathrm{~b}$, respectively. Low and high resolution SEM images reflect not only the homogeneous globular morphology typically observed for p-doped PEDOT, but also the existence of multiple and tortuous pores. The diameter of these pores, which are defined by thin sticks that connect dense clusters of polymer chains (Figure $2 \mathrm{a}$ ), is variable, ranging from 0.4 to $2.0 \mu \mathrm{m}$. Application of a potential of -1.30 $\mathrm{V}$ results in a drastic reduction of the porosity, even though the surface retains the 
globular appearance (Figure 2b). More specifically, the applied reduction potential induces the partial collapse of the clusters, which is probably caused by the breakage of some of the above mentioned sticks (i.e. the thinner ones). Consequently, the shape of pores becomes less defined and their diameter rarely exceeds $\sim 1.1 \mu \mathrm{m}$. Height AFM images displayed in Figures 2c-d are fully consistent with SEM micrographs, evidencing that PEDOT clusters approach after the dedoping process. The roughness of the sample increases from $R_{q}=600-700 \mathrm{~nm}$ for $\mathrm{p}$-doped PEDOT to $\mathrm{R}_{\mathrm{q}}=720-850 \mathrm{~nm}$ for the dedoped CP.

\section{Redoping of PEDOT films with $m$ PI for n-doping}

The electrochemical reduction (n-doping) was performed by chronoamperometry applying a constant reduction potential. In order to determine the most suitable value, reduction potentials of $-0.50,-0.70,-0.90$ and $-1.10 \mathrm{~V}$ were applied during $150 \mathrm{~s}$ to dedoped PEDOT films in $10 \mathrm{mM} m \mathrm{PI}$ aqueous solutions, whereas control assays (blank) were performed using identical conditions but in absence of PEDOT (i.e. using bare steel as working electrode). Chronoamperograms recorded for the different reduction potentials, which are displayed in Figure 3a, show that the current density of n-doped PEDOT films stabilizes at a lower value when more negative is potential, evidencing that the success of the redoping process increases from -0.50 to $-1.10 \mathrm{~V}$. The potential also affects the blank assays, even though the current density stabilizes at values significantly higher than those reached in redoping experiments. This feature is clearly reflected in Figure 3b, which compares the current density for the redoping and blank assays reached after $150 \mathrm{~s}$.

Another important difference between the chronoamperograms recorded for redoping and blank assays is the time required by current density to reach the steady state. 
Independently of the reduction potential, this time is very short for blank assays since the diffusion rate of the $m$ PI molecules through the electrochemical solution is very fast. In contrast, the stabilization time in redoping experiments, which is considerably higher than for the blank assays, increases with decreasing reduction potential. This behavior evidences that the number of $m$ PI molecules entering into the PEDOT film grows with decreasing reduction potential and, also, that the movement of the dopant molecules is slower into the CP matrix than in the electrochemical solution.

For each reduction potential, the neat redoping charge $\left(Q_{r d o p}^{\text {neat }}\right)$ was estimated as the difference between the chronoamperometric charge obtained for the redoped PEDOT film $\left(Q_{\text {rdop }}\right)$ and the chronoamperometric charge derived for the corresponding blank $\left(Q_{\text {blank }}\right)$ :

$$
Q_{r d o p}^{\text {neat }}=Q_{\text {rdop }}-Q_{\text {blank }}
$$

Figure 3c compares the values of $Q_{r d o p}$ and $Q_{r d o p}^{\text {neat }}$ for the four used reduction potentials. The value of $Q_{r d o p}^{\text {neat}}$, which indicates the charge for PEDOT averaged by repeat unit after the incorporation of $m$ PI polycations, becomes one order of magnitude more negative when the reduction potential decreases to $-1.10 \mathrm{~V}$. The values of $Q_{r d o p}^{\text {neat }}$ were used to estimate moles of ionene repeating units $\left(N_{m P I}\right)$ incorporated during the redoping process for each reduction potential (Table 1):

$$
N_{m P I}=\frac{Q_{r d o p}^{\text {neat }}}{2 F}
$$

where $\mathrm{F}$ is the Faraday's constant and include two positive charges of every $m \mathrm{PI}$ repeat unit. The moles of EDOT repeat units incorporated to the film during the polymerization process $\left(N_{E D O T}\right)$ and the moles of $\mathrm{ClO}_{4}^{-}\left(N_{C l O 4}\right)$ escaping from the film during the dedoping process were determined using similar expressions: 


$$
\begin{aligned}
& N_{\text {EDOT }}=\frac{\left(Q_{\text {pol }}-Q_{\text {ddop }}\right)}{2 F} \\
& N_{\text {ClO4- }}=\frac{Q_{d d o p}}{1 F}
\end{aligned}
$$

where $Q_{p o l}$ is the charge consumed during the polymerization process of $\mathrm{p}$-doped PEDOT films and $Q_{d d o p}$ is the charge consumed during the dedoping process. As $Q_{p o l}$ was fixed at $0.960 \mathrm{C}$, the value derived from was $N_{E D O T}=4.26 \cdot 10^{-6}$ moles of EDOT (Eqn 3). The values of $Q_{d d o p}$ and $N_{C l O 4-}$ depended not only on the reduction potential ($1.30 \mathrm{~V}$ ) but also on the structure of the sample. Average values of $Q_{d d o p}$ and $N_{\text {ClO4- }}$ (Eqn 4) for the set samples used for each redoping potential, which are relatively similar for all sets, are included in Table 1. Finally, the doping level, which expresses the fraction of $m$ PI unit per EDOT unit, was derived from:

$$
d l_{m P I}=\frac{N_{m P I}}{N_{E D O T}}
$$

The values of $d l_{m P I}$ for each reduction potential (Table 1) indicates one dicationic $m$ PI unit for every $\sim 16, \sim 48, \sim 45$ and $\sim 67$ EDOT units when the reduction potential is $1.10,-0.90,-0.70$ and $-0.50 \mathrm{~V}$, respectively. Unfortunately, the redoping process is limited by the electrolysis of water into oxygen and hydrogen gas, which occurs at $1.23 \mathrm{~V}$, precluding the use of lower reduction potentials.

The successful incorporation of $m \mathrm{PI}$ has been proved by X-ray photoelectron spectroscopy (XPS). Table 2 compares the atomic percent compositions of p-doped PEDOT, dedoped PEDOT, and PEDOT redoped with $m$ PI using a potential of $-1.10 \mathrm{~V}$. As it can be seen, the C / S ratio is similar (5.6) and close to the expected value (6.0) for both p-doped and dedoped PEDOT. In contrast, the O / S decreases from 2.8 (p-doped) to 2.3 (dedoped), reflecting the success of the dedoping process. The C / S and O / S ratios for PEDOT redoped with $m \mathrm{PI}$ are 12.1 and 2.7, which are far from the values 
expected to PEDOT and, therefore, corroborate the incorporation of the doping agent. However, the most noticeable result is the apparition of nitrogen that unequivocally proves the incorporation of $m \mathrm{PI}$.

Figure 4 compares the surface morphology of samples redoped using -1.10 and $0.50 \mathrm{~V}$ reduction potentials. As it can be seen, the $-1.10 \mathrm{~V}$ potential does not induce changes in the CP morphology (Figure 4a), which preserves the globular appearance and porosity of the dedoped sample (Figure $2 \mathrm{~b}$ ). However, nanostructured $m \mathrm{PI}$ is clearly observed at the surface of the samples. Thus, this polycation frequently organizes in nanospheres with diameters ranging from 25 to $75 \mathrm{~nm}$ (Figure 4a, right), even though ultrathin sheets are also eventually detected (Figure 4a, middle). These results are consistent with the relatively high doping level obtained at such potential (i.e. one dicationic $m$ PI unit every 16 EDOT units. In opposition, the highest reduction potential $(-0.50 \mathrm{~V})$ severely affects the surface morphology of PEDOT films (Figure $4 \mathrm{~b})$. Thus, CP aggregates undergo a drastic shrink, reducing their diameter more than a half. Although the porosity is retained after such shrinking process (Figure $4 \mathrm{~b}$, right), the diffusion of $m \mathrm{PI}$ chains at $-0.50 \mathrm{~V}$ is very slow, limiting their entrance into the $\mathrm{CP}$ matrix (i.e. one dicationic mPI unit every $\sim 67$ EDOT units). In contrast, this potential is enough to expel solvent molecules and residual perchlorate anions embedded into the CP clusters, causing the observed shrinking phenomenon.

Height and phase AFM images (Figures 5a-b) corroborate SEM observations. In particular, phase imaging, which is sensitive to surface stiffness/softness, allows chemical mapping of surfaces based on material differences. Phase shifts, which are registered as bright and dark regions in phase images, are more abundant and contrasted for samples redoped at $-1.10 \mathrm{~V}$ than at $-0.50 \mathrm{~V}$. This observation is consistent with the nanorganization of the $m$ PI molecules discussed above. Indeed, the structure obtained 
for $\mathrm{n}$-doped samples obtained at $-1.10 \mathrm{~V}$ is pretty similar to the nanosegregated phase distribution observed for PEDOT with PSS, ${ }^{13}$ evidencing that polymeric dopant agents provide significant differences with respect to small dopants. Moreover, the root-meansquare roughness $\left(\mathrm{R}_{\mathrm{q}}\right)$ of samples obtained using $-1.10 \mathrm{~V}$ as redoping potential is significantly higher than that of samples redoped using-0.50 V (Figures 5a-b).

\section{Comparison between PEDOT n-doped with $m$ PI and TMA}

PEDOT films redoped with TMA were obtained as described in the previous subsection for $m$ PI. More specifically, dedoped PEDOT films were electrochemically reduced by chronoamperometry applying a constant reduction potential of -0.50 or $1.10 \mathrm{~V}$ during $150 \mathrm{~s}$ in $10 \mathrm{mM}$ TMA aqueous solutions. The successful incorporation of TMA is proved by XPS, the elemental composition of the films redoped using a potential of $-1.10 \mathrm{~V}$ being included in Table 2 . Thus, nitrogen is successfully detected in PEDOT samples redoped with TMA. The doping level, expressed as the fraction of TMA molecules per EDOT unit, was calculated using Eqn 5 but replacing $N_{m P I}$ by $N_{T M A}$, which corresponds to the moles of TMA incorporated during the redoping process:

$$
N_{T M A}=\frac{Q_{r d o p}^{\text {neat }}}{1 F}
$$

The doping level $\left(d l_{T M A}\right)$, expressed as the fraction of TMA molecules per EDOT, $Q_{\text {rdop }}^{\text {neat }}$ and $N_{T M A}$ average values are included in Table 1 . Considering that a TMA molecule and an $m \mathrm{PI}$ repeat unit involve one and two positive charges, respectively, comparison of the $d l_{T M A}$ and $d l_{m P I}$ indicates similar qualitative behaviors for the two ndopant anions. However, the mobility of small TMA molecules is higher than that of $m$ PI chains and, therefore, the influence of the reduction potential in $d l_{T M A}$ is more 
important than in $d l_{m P I}$. Thus, the amount of negative charge incorporated by PEDOT repeat unit is higher by more twice for TMA. More specifically, one cationic TMA molecule has been incorporated every $\sim 31$ and $\sim 11$ EDOT units when the redoping potential is -1.10 and $-0.50 \mathrm{~V}$, respectively.

SEM micrographs and AFM images recorded form PEDOT samples redoped with TMA (Figures 4c-d and 5c-d, respectively) display features, in general, similar to those discussed above for samples redoped with $m \mathrm{PI}$ (Figures $4 \mathrm{a}-\mathrm{b}$ and 5a-b), even though the apparition of cation organized nanostructures when the reduction potential is $-1.10 \mathrm{~V}$ is much less pronounced for TMA than for $m$ PI. Indeed, TMA nanospheres are not only less abundant but also smaller than $m$ PI nanospheres (Figures $4 \mathrm{~d}$ and $4 \mathrm{a}$, respectively).

\section{Computer simulations}

Atomistic molecular dynamics (MD) simulations were performed considering 48 PEDOT and $32 \mathrm{mPI}$ chains, each one consisting of 40 and 6 repeat units, respectively (i.e. $48 \times 40=1920$ EDOT repeat units and $32 \times 6=192$ ionene repeat units). Although recent computational advances allow the study of relatively large polymeric systems, as the one studied in this work, their size is still limited in comparison with the experimental one. In order to overcome this limitation, the doping level of the simulated model has been increased with respect to that electrochemically measured (Table 1). More specifically, a charge of -1 was accounted for every five EDOT units, while a total charge of +2 was considered for each ionene repeat unit (i.e. the model contains one dicationic $m$ PI unit for every 10 EDOT units rather than for every 16 , as observed).

Computer simulations were performed considering two different models, hereafter M1 and M2, which differ in the initial disposition of PEDOT and $m$ PI chains. In M1 both $m$ PI and PEDOT chains were regularly arranged (i.e. each $m$ PI chain was 
surrounded by three PEDOT chains), while in $\mathbf{M} 2$ the polymeric chains of the two species were distributed in two groups that in turn were arranged in separated blocks. Figures $6 \mathrm{a}$ and $6 \mathrm{~b}$ depict the starting points used for MD simulations. The density was $\rho=1.0 \mathrm{~g} / \mathrm{cm}^{3}$ for both initial models, which is much lower than the experimentally determined value (see below).

After equilibration of the starting geometries, as is described in the Supplementary Information, productive trajectories of $75 \mathrm{~ns}$ NPT-MD were run at room temperature for both M1 and M2 models. PEDOT and $m$ PI chains underwent significant rearrangements during the first period of the two simulations ( $\sim 15 \mathrm{~ns})$, especially M2, while models remained practically unaltered during the last 25 ns (i.e. only local conformations changes were observed). Both M1 and M2 stabilized at same density, $\rho=1.44 \mathrm{~g} / \mathrm{cm}^{3}$, indicating that polymer chains approached during the simulations (i.e. compression behavior). The experimental density of PEDOT samples redoped with $m$ PI using a reduction potential of $-1.10 \mathrm{~V}$ was estimated using the flotation method (see Supplementary Information). The resulting value, $\rho=1.55 \mathrm{~g} / \mathrm{cm}^{3}$, differs from the theoretical prediction by $7.6 \%$ only, evidencing that, despite the artificial increase of doping level introduced in the polymeric models, MD simulations successfully capture polymer $\cdots$ polymer interactions. The structures obtained for the two models at the end of the production trajectories are included in Figures 6a and 6b. Although M1 is enthalpically favored (i.e. negatively charged PEDOT chains and cationic $m \mathrm{PI}$ are individually confronted and, therefore, repulsive interactions between chains with the same charge are avoided), the preferred model is M2. Thus, the entropic contribution is significantly more favorable for disordered M2 than for the highly ordered M1, compensating the enthalpic contribution. 
Although M1 is dominated by electrostatic PEDOT $\cdots m$ PI interactions, $\pi-\pi$ stacking interactions are expected to play a crucial role in M2 where PEDOT...PEDOT and $m \mathrm{PI} \cdots m \mathrm{PI}$ interactions are more abundant. Figure $6 \mathrm{c}$ represents the partial radial distribution function for pairs of centers of masses of aromatic rings belonging to different chains, $g_{a r-a r}$. The intense and relatively broad peak centered at $r \approx 4.5 \AA$ for M2 demonstrates the importance of the $\pi-\pi$ stacking in the stability of this model. Besides, M2 shows two less intense peaks centered at $r=11.5$ and $18.4 \AA$, which reflect the local order induced by the all-anti conformation of the negatively charge PEDOT chains. On the other hand, the peak at $r \approx 4.5 \AA$ is much less intense for M1 indicating that $\pi-\pi$ stacking interactions between aromatic rings located at neighboring PEDOT and $m \mathrm{PI}$ chains are less abundant than in $\mathbf{M 2}$ (i.e. the total number of $\pi-\pi$ interactions is around 2.5 higher for $\mathbf{M} 2$ than for $\mathbf{M 1}$ when a cutoff distance of $5.0 \AA$ is used). Consequently, the peaks at $r>10 \AA$ attributed to the polymer chains periodicity are imperceptible or inexistent in M1.

The broadness of the peak identified at $r \approx 4.5 \AA$ for $\mathbf{M 2}$, which extends from 3.8 to $5.3 \AA$ (Figure 6c), is compatible with the co-existence of $\pi-\pi$ stacking interactions in which the two aromatic rings are coplanar (sandwich configuration) or perpendicular (T-shaped configuration). Previous theoretical studies based on sophisticated quantum mechanical methods evidenced that the center of masses of two interacting thiophene or benzene aromatic rings are ideally separated by $\sim 3.7 \AA$ and $\sim 5.0 \AA$ for the sandwich and T-shaped configuration, respectively. ${ }^{37-39}$ In order to examine more exhaustively this hypothesis, the angle $(\theta)$ formed by aromatic rings involved in the $\pi$ - $\pi$ stacking interactions of M1 and M2 was evaluated. Specifically, $\theta$ was calculated for all pairs of ring belonging to different polymer chains and with the centers of masses separated by $\leq 5.5 \AA$. This angle is $\theta \leq 45^{\circ}$ for the sandwich configuration (ideally, $\theta=0^{\circ}$ ), whereas 
the T-shaped configuration corresponds to angles comprised between $45^{\circ}$ and $90^{\circ}$ (ideally, $\theta=90^{\circ}$ ).

The distribution of $\pi-\pi$ stacking interactions is displayed in Figure $6 \mathrm{~d}$ for both M1 and M2. The two models show a clear preference towards the sandwich configuration (78\%), even though $\theta$ deviates considerably from the ideal value. Thus, the two interacting rings exhibit some tilting, as is evidenced by both the broad distribution profile and the peaks appearing in the interval comprised between $15^{\circ}$ and $30^{\circ}$. Similarly, the small fraction of aromatic ring interacting through the T-shaped configuration displays important distortions with respect to the ideal value of $\theta=90^{\circ}$, the latter exhibiting a very low population.

\section{Effect of the dopant in the surface wettability and the thermal stability}

The very different chemical nature of $m \mathrm{PI}$ and TMA dopant anions is expected to affect the properties of the CP. In this work we have compared the influence of the dopants in the wettability and the thermal stability of redoped PEDOT by measuring the contact angle $(\theta)$ in water and by thermogravimetry (TGA), respectively.

Wettability is related with both surface topography, the chemical nature of the surface and the doping level. Oxidized PEDOT is a hydrophilic material with $\theta=$ $65^{\circ} \pm 6^{\circ}$ (Figure $7 \mathrm{a}$ ), which should be attributed to the oxygen atoms of the fused dioxane ring and the positive charge delocalized through the polymer chains. Amazingly, the reduction process increases considerably the hydrophilicity of PEDOT $\left(\theta=37^{\circ} \pm 5^{\circ}\right)$. Thus, although reduced polymer chains are expected to exhibit less affinity by water than oxidized polymer chains, the drastic reduction of the surface roughness from submicrometric to nanometric scale (Figure 5) causes the opposite effect. 
Re-doping with TMA results in a hydrophobic surface, $\theta=101^{\circ} \pm 12^{\circ}$, which has been attributed to the TMA nanostructures observed at the surface of the films (Figure 4c). Both the accumulated exposition of hydrophobic methyl groups arising from those superficial nanostructures and the shielding of the positively charged nitrogen atom due to the tetrahedron geometry of TMA molecules drastically reduce the affinity towards water. Besides, the surface roughness of PEDOT redoped with TMA is intermediate between those of the oxidized and reduced CP. In opposition, PEDOT films redoped with $m$ PI results in hydrophilic surfaces that, independently of the redoping potential, exhibit higher affinity by water than p-doped PEDOT. Indeed, the wettability of redoped PEDOT is comparable to that of the reduced polymer. This cannot be associated to the low doping level of PEDOT redoped with $m$ PI, which is comparable to that obtained with TMA (Table 1), nor to the surface roughness, which is similar to that of p-doped PEDOT (Figure 5). Indeed, the hydrophilic character of PEDOT redoped with $m$ PI has been attributed to the accessibility of the polar amide groups of the polycation, which facilitate the formation of hydrogen bonding interactions with water molecules at the film/drop interface.

Thermogravimetric analyses (Figure $7 b$ ) reflect the significant influence of the dopant agent in the thermal stability of n-doped PEDOT. First, differential thermogravimetric analysis (DGTA) curves of dedoped and redoped PEDOT show that solvent evaporation starts at $\sim 45^{\circ}$. After this, a pronounced degradation step is observed for all systems. The maximum temperature $\left(\mathrm{T}_{\max }\right)$ for this degradation is around $15^{\circ} \mathrm{C}$ lower for dedoped PEDOT $\left(\mathrm{T}_{\max }=338^{\circ} \mathrm{C}\right)$ than for redoped PEDOT $\left(\mathrm{T}_{\max }=352{ }^{\circ} \mathrm{C}\right)$, independently of the dopant. However, the temperature at a $50 \%$ weight loss $\left(\mathrm{T}_{50 \%}\right)$ increases as follows: dedoped PEDOT $\left(\mathrm{T}_{50 \%}=371{ }^{\circ} \mathrm{C}\right)<$ PEDOT redoped with TMA $\left(\mathrm{T}_{50 \%}=403{ }^{\circ} \mathrm{C}\right)<$ PEDOT redoped with $m \mathrm{PI}\left(\mathrm{T}_{50 \%}=427{ }^{\circ} \mathrm{C}\right)$. Moreover, these 
differences are preserved at a $70 \%$ weight loss $\left(\mathrm{T}_{70 \%}\right)$ with values of 449,465 and 518 ${ }^{\circ} \mathrm{C}$, respectively. Overall, the profiles displayed in Figure $7 \mathrm{~b}$ show that the ionene enhances the thermal stability of the n-doped $\mathrm{CP}$ with respect to conventional small organic molecules, like TMA. This has been attributed to the fact that PEDOT $\cdots m$ PI interactions are stronger than PEDOT $\cdots$ TMA due to both the polymeric nature and the presence of polar amide groups in $m \mathrm{PI}$ chains.

\section{CONCLUSIONS}

Our studies on the properties of redoped PEDOT have demonstrated for the first time that ionenes can be successfully used as n-dopant agents, offering an important alternative to conventional TMA and its, also small, derivatives. The highest doping level has been achieved applying a redoping potential of $-1.10 \mathrm{~V}$ to a PEDOT film, which was previously dedoped at a fixed potential of $-1.30 \mathrm{~V}$, in an aqueous solution of $m$ PI. SEM and AFM analyses reveal some segregation between the PEDOT matrix, which retain the typical globular and porous morphology, and the ionene that organization into nanospheres and ultrathin sheets. This particular organization is consistent with results derived from atomistic MD simulations, which show that PEDOT $\cdots$ PEDOT and $m$ PI $\cdots m$ PI interactions predominate over PEDOT $\cdots m$ PI interactions. On the other hand, the doping level achieved for PEDOT films n-doped with $m \mathrm{PI}$ is similar to that obtained using TMA, even though properties of the material are significantly different. More specifically, the incorporation of $m$ PI results in a significant increment of the hydrophilicity and the thermal stability in comparison to pdoped and dedoped PEDOT, respectively. In contrast, TMA converts the film into hydrophobic and the thermal stability is intermediate between those of films dedoped and redoped with $m$ PI. Overall, these results indicate that redoping with ionenes can be 
a successful strategy to produce n-doped PEDOT electrodes with properties very different from those achieved using small organic cations.

\section{ACKNOWLEDGEMENTS}

Authors acknowledge MINECO/FEDER (MAT2015-69367-R) for financial support. M.S.G is grateful to Costa Rica National Commission of Scientific and Technological Research (CONICYT-Support $\mathrm{N}^{\circ}$ FI-172B-14). Support for the research of C.A. was received through the prize "ICREA Academia" for excellence in research funded by the Generalitat de Catalunya. D.D.D. the Deutsche Forschungsgemeinschaft (DFG) for the Heisenberg Professorship Award.

\section{REFERENCES}

1. C. Lei, P. Wilson and C. Lekakou, J. Power Sources, 2011, 196, 7823-7827.

2. K. S. Ryu, Y. G. Lee, Y. S. Hong, Y.J.Park, X. L. Wu, K. M. Kim, M. G. Kang, N. G. Park and S. H. Chang, Electrochim. Acta, 2004, 50,843-847.

3. L. B. Groenendaal, F. Jonas, D. Freitag, H. Pielartzik and J. R. Reynolds, Adv. Mater., 2000, 12, 481-494.

4. D. Aradilla, F. Estrany and C. Alemán, J. Phys. Chem. C, 2011, 115, 8430-8438.

5. Q. Wei, M. Mukaida, K. Kirihara, Y. Naitoh and T. Ishida, Materials, 2015, 8, $732-750$.

6. J. Poater, J. Casanovas, M. Solà and C. Alemán, J. Phys. Chem. A, 2010, 114, $1023-1028$.

7. W. Plieth, A. Bund, U. Rammelt, S. Neudeck and L. M. Duc, Electrochim. Acta, 2006, 51, 2366-2372. 
8. C. Ocampo, R. Oliver, E. Armelin, C. Alemán and F. Estrany, J. Polym. Res., 2006, 13, 193-200.

9. A. Bund and S. Neudeck, J. Phys. Chem. B, 2004, 108, 17845-17850.

10. C. Li and T. Imae, Macromolecules, 2004, 37, 2411-2416.

11. J. Huang, P. F. Miller, J. S. Wilson, A. J. de Mello, J. C. de Mello and D. D. Bradley, Adv. Funct. Mater., 2005, 15, 290-296.

12. A. M. Nardes, M. Kemerink, M. De Kok, E. Vinken, K. Maturova and R. Janssen, Org. Electron., 2008, 9, 727-734.

13. U. Lang, N. Naujoks and J. Dual, Synth. Met., 2009, 159, 473-479.

14. T. Stöcker, A. Köhler and R. Moos, J. Polym. Sci., Part B: Polym. Phys., 2012, 50, 976-983.

15. M. Kuş and S. Okur, Sens. Actuators B, 2009, 143, 177-181.

16. Y. Jiang, B. Luo, F. Jiang, F. Jiang, C. Fuentes-Hernandez, T. Liu, L. Mao, S. Xiong, Z. Li and T. Wang, Nano Lett., 2016, 16, 7829-7835.

17. J. Tong, S. Xiong, Y. Zhou, L. Mao, X. Min, Z. Li, F. Jiang, W. Meng, F. Qin and T. Liu, Mater. Horiz., 2016, 3, 452-459.

18. H. Okuzaki, T. Kuwabara, K. Funasaka and T. Saido, Adv. Funct. Mater., 2013, 23, 4400-4407.

19. S. Taccola, F. Greco, E. Sinibaldi, A. Mondini, B. Mazzolai and V. Mattoli, $A d v$. Mater., 2015, 27, 1668-1675.

20. Y. Li, M. Liu, Y. Li, K. Yuan, L. Xu, W. Yu, R. Chen, X. Qiu and H.-L. Yip, $A d v$. Energy Mater., 2017, 7, 1601499.

21. Q.B. Pei, G. Zuccarello, M. Ahlskog and O. Inganäs, Polymer, 1994, 35, $1347-1351$. 
22. C. Kvarnström, H. Neugebauer, A. Ivaska and N. S. Sariciftci, J. Mol. Struct., 2000, 521, 271-277.

23. H. J. Ahonen, J. Lukkari and J. Kankare, Macromolecules, 2000, 33, 6787-6793.

24. M. Skompska, J. Mieczkowski, R. Holze and J. Heinze, J. Electroanal. Chem., 2005, 577, 9-17.

25. A. R. Hillman, S. J. Daisley and S. Bruckenstein, Electrochim. Acta, 2008, 53, $3763-3771$.

26. H. Gustafsson, C. Kwarnström and A. Ivaska, Thin Solid Films, 2008, 517, 474478.

27. A. P. Sandoval, J. M. Feliu, R. Torresi and M. F. Suárez, RSC Adv., 2014, 4, $3383-$ 3391.

28. V. D. Pokhodenko, V. A. Krylov and N. V. Konoshchuk, Synth. Met., 1999, 99, $91-95$.

29. V. D. Pokhodenko and V. A. Krylov, Synth. Met., 1991, 41, 533-536.

30. M. Mastragostino and L. Soddu, Electrochim. Acta, 1990, 35, 463-466.

31. W. Jaeger, J. Bohrisch and A. Laschewsky, Prog. Polym. Sci., 2010, 35, 511-586.

32. J. K. Oha, R. Drumright, D. J. Siegwart and K. Matyjaszewski, Prog. Polym. Sci., $2008,33,448-477$.

33. M. R. Tant, K. A. Mauritz and G. L. Wilkes, Ionomers: Synthesis, Structure, Properties, and Applications, Blackie Academic and Professional, London, 1997.

34. J. Bachl, D. Zanuy, D. E. López-Pérez, G. Revilla-López, C. Cativiela, C. Alemán and D. Díaz Díaz, Adv. Funct. Mater., 2014, 24, 4893-4904.

35. J. Bachl, O. Bertran, J. Mayr, G. C. Alemán and D. Díaz Díaz, Soft Matter, 2017, 13, 3031-3041. 
36. C. Ocampo, R. Oliver, E. Armelin, C. Alemán and F. Estrany, J. Polym. Res., 2006, 13, 193-200.

37. S. Tsuzuki, K. Honda, T. Uchimaru, M. Mikami and K. Tanabe, J. Am. Chem. Soc., 2002, 124, 104-112.

38. F. Rodriguez-Ropero, J. Casanovas and C. Alemán, J. Comput. Chem., 2008, 29, 69-78.

39. S. Tsuzuki, K. Honda and R. Azumi, J. Am. Chem. Soc., 2002, 124, 12200-12209. 
Table 1. Neat redoping charge ( $Q_{r d o p}^{\text {neat }}$; Eqn 1), moles of ionene repeating units incorporated during the redoping process $\left(N_{m P I}\right), Q_{d d o p}$ is the charge consumed during the de-doping process $\left(Q_{d d o p}\right.$; Eqn 3), moles of $\mathrm{ClO}_{4}^{-}$escaping from the film during the dedoping process $\left(\mathrm{N}_{\mathrm{ClO} 4}\right)$, and doping level expressed as the fraction of dicationic $\mathrm{mPI}$ unit per EDOT unit $\left(d l_{m P I}\right.$; Eqn 5).

\begin{tabular}{lccccc}
\hline Reduction potential (V) & $Q_{\text {rdop }}^{\text {neat }}$ & $N_{m P I}$ & $Q_{d d o p}$ & $N_{C l O 4-}$ & $d l_{m P I}$ \\
\hline-0.50 & -0.0110 & $5.68 \cdot 10^{-8}$ & -0.1267 & $1.31 \cdot 10^{-6}$ & 0.015 \\
-0.70 & -0.0151 & $7.80 \cdot 10^{-8}$ & -0.1382 & $1.43 \cdot 10^{-6}$ & 0.022 \\
-0.90 & -0.0166 & $8.59 \cdot 10^{-8}$ & -0.1345 & $1.39 \cdot 10^{-6}$ & 0.021 \\
-1.10 & -0.0398 & $2.06 \cdot 10^{-7}$ & -0.1605 & $1.66 \cdot 10^{-6}$ & 0.063 \\
& & & & & \\
& $Q_{\text {rdop }}^{\text {neat }}$ & $N_{T M A}$ & $Q_{d d o p}$ & $N_{C l O 4-}$ & $d l_{T M A}$ \\
-0.50 & -0.0127 & $1.32 \cdot 10^{-7}$ & -0.0820 & $8.50 \cdot 10^{-7}$ & 0.032 \\
-1.10 & -0.0272 & $2.82 \cdot 10^{-7}$ & -0.1749 & $1.81 \cdot 10^{-6}$ & 0.089 \\
\hline
\end{tabular}


Table 2. Atomic percent composition ( $\mathrm{C} 1 \mathrm{~s}, \mathrm{~N}$ 1s, O 1s and $\mathrm{S} 2 \mathrm{p}$ ) obtained by XPS for PEDOT: p-doped, dedoped, and redoped (n-doped) with $m$ PI or TMA

\begin{tabular}{ccccc}
\hline Sample & C 1s & N 1s & O 1s & S 2p \\
\hline p-doped & 59.2 & 0.0 & 30.2 & 10.6 \\
Dedoped & 63.3 & 0.0 & 25.5 & 11.2 \\
n-doped TMA & 64.6 & 0.5 & 23.8 & 11.1 \\
n-doped $m$ PI & 72.8 & 4.9 & 16.3 & 6.0 \\
\hline
\end{tabular}




\section{CAPTIONS TO FIGURES}

Figure 1. (a) Chronopotentiometric time-potential profile obtained for p-doped PEDOT at $-1.0 \mathrm{~mA}$ in water with $10 \mathrm{mM} \mathrm{LiClO}_{4}$. (b) Chronopotentiometric dedoping for p-doped PEDOT in water with $10 \mathrm{mM} \mathrm{LiClO}_{4}$ applying $-1.0 \mathrm{~mA}$ during $150 \mathrm{~s}$ two consecutive times (first and second processes). (c) Charge consumed during the chronoamperometric dedoping $\left(Q_{d d o p}\right.$, in C) at a fixed potential (from -1.10 to $-1.60 \mathrm{~V}$ ) during $100 \mathrm{~s}$ in a $10 \mathrm{mM} \mathrm{LiClO}_{4}$ water solution.

Figure 2. Low and high resolution SEM images (left and right, respectively) of: (a) p-doped PEDOT prepared in acetonitrile with $100 \mathrm{mM} \mathrm{LiClO}_{4}$ at a constant potential of $+1.40 \mathrm{~V}$; and (b) Dedoped PEDOT in water with $10 \mathrm{mM} \mathrm{LiClO}_{4}$ at a constant potential of $-1.30 \mathrm{~V}$. Height AFM images $\left(5 \times 5 \mu \mathrm{m}^{2}\right)$ of (c) p-doped and (d) dedoped PEDOT are also displayed.

Figure 3. Chronopotentiometric time-current density $(j)$ profiles obtained for the redoping of PEDOT films and blank assays (see text) in water with $10 \mathrm{mM} m \mathrm{mI}$ using different reduction potentials. (b) Variation of the current density reached after $150 \mathrm{~s}$ against the reduction potential for the redoping and blank assays. (c) Variation of the redoping charge and the neat redoping charge (Eqn 1) against the reduction potential. (d)

Figure 4. Low and high resolution (left and right, respectively) SEM micrographs of PEDOT films redoped with $(\mathrm{a}, \mathrm{b}) m \mathrm{PI}$ and $(\mathrm{c}, \mathrm{d})$ TMA using a reduction potential of $(\mathrm{a}$, c) $-1.10 \mathrm{~V}$ or $(\mathrm{b}, \mathrm{d})-0.50 \mathrm{~V}$. The blue square and the red circles in (a) highlight the presence of $m$ PI organized in ultrathin sheets (eventually observed) and nanospheres (frequently observed), respectively. Nanospheres are also detected in (c).

Figure 5. Height and phase (left and right, respectively) AFM images of PEDOT films redoped with $(\mathrm{a}, \mathrm{b}) \mathrm{mPI}$ and $(\mathrm{c}, \mathrm{d})$ TMA using a reduction potential of $(\mathrm{a}, \mathrm{c})-1.10$ 
V or $(b, d)-0.50 \mathrm{~V}$. Representative cross-sectional profiles showing the topography and the root-mean-square roughness $\left(\mathrm{R}_{\mathrm{q}}\right)$ values are also displayed.

Figure 6. Initial geometry (left) and last snapshot from the productive NPT-MD trajectory (right) for (a) M1 and (b) M2 models. (c) Partial radial distribution functions for the pairs of centres of masses of aromatic rings belonging to different polymer chains for both M1 and M2. (d) Distribution of the number of pairs of interacting aromatic rings as a function of the angle $\theta$ formed by such planar rings for both $\mathbf{M 1}$ and M2. Only ring belonging to different polymer chains and with the centers of masses separated by $\leq 5.5 \AA$ h have been considered.

Figure 7. (a) Contact angle of water measured for oxidized (p-doped) PEDOT, PEDOT reduced by chronoamperometry at constant potential of $-1.30 \mathrm{~V}$, PEDOT redoped with $m \mathrm{PI}$ using a reduction potential of $-0.50,-0.70,-0.90$ and $-1.10 \mathrm{~V}$, and PEDOT redoped with TMA using a reduction potential of $-1.10 \mathrm{~V}$. (b) TGA and DTGA (inset) curves of PEDOT reduced at constant potential of $-1.30 \mathrm{~V}$, and PEDOT redoped with TMA or $m$ PI using a reduction potential of $-1.10 \mathrm{~V}$. 
(a)
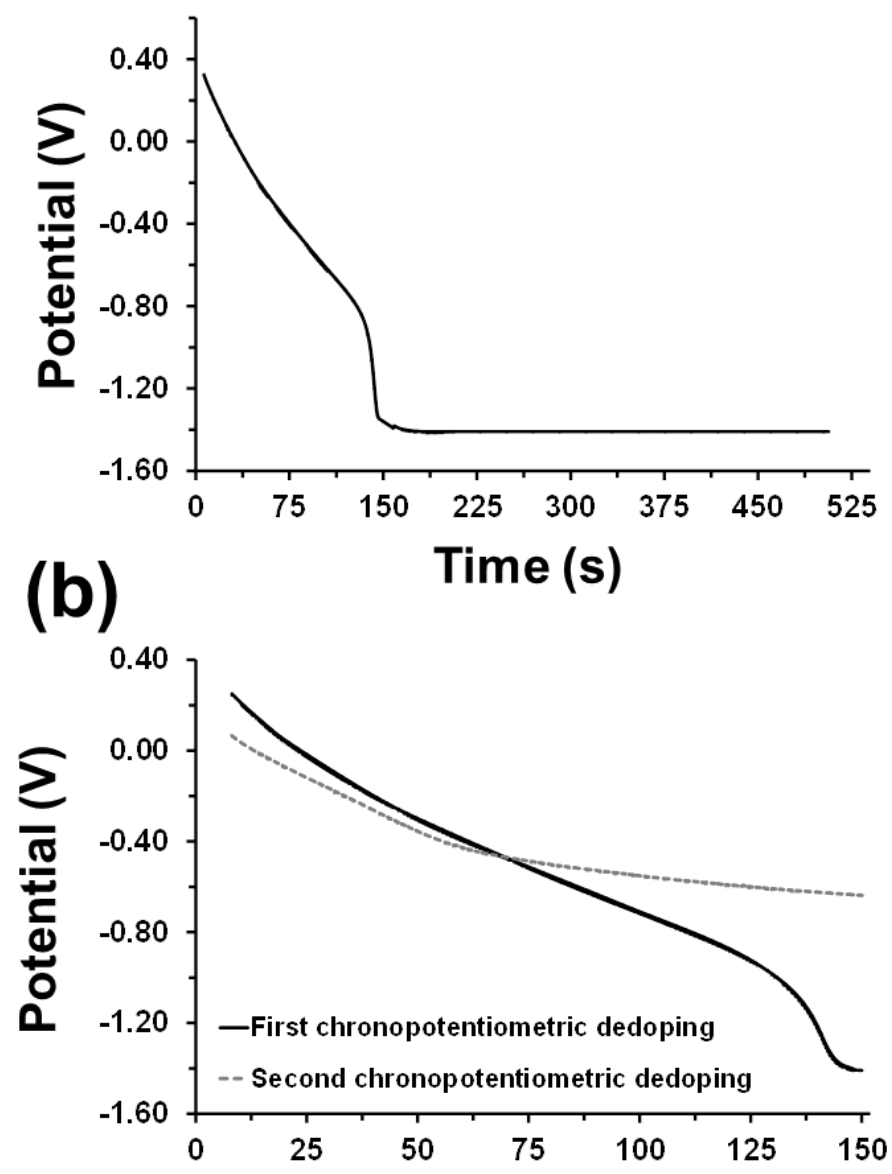

(c)

Time (s)

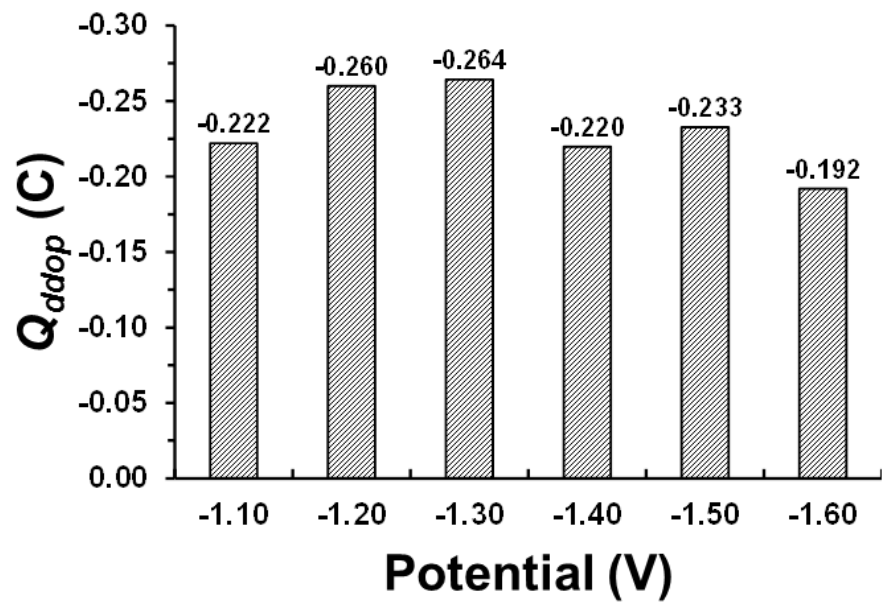

Figure 1 

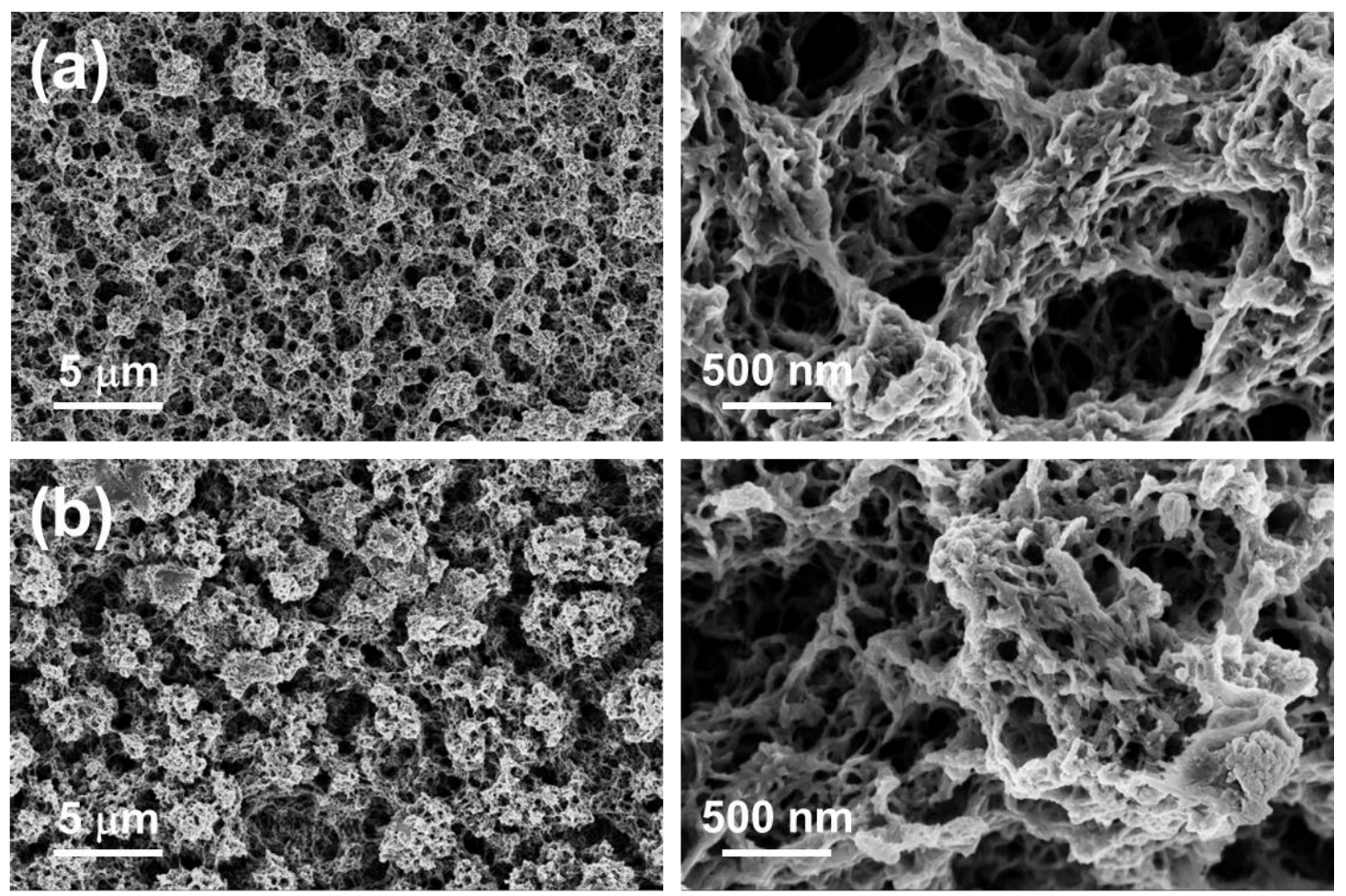

(c)

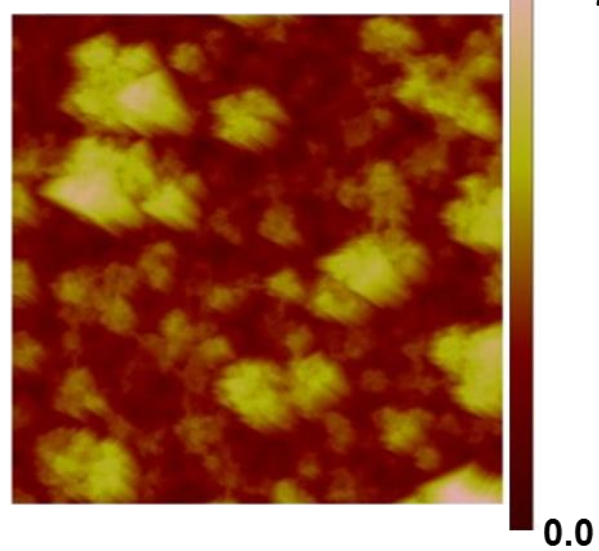

(d)

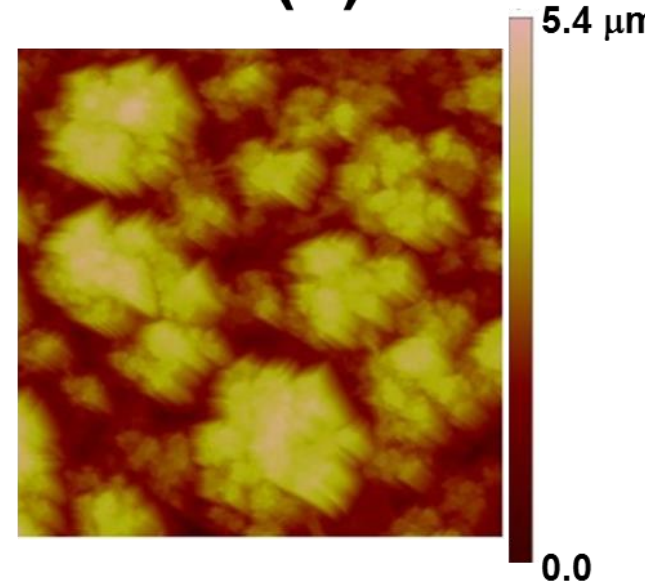

Figure 2 

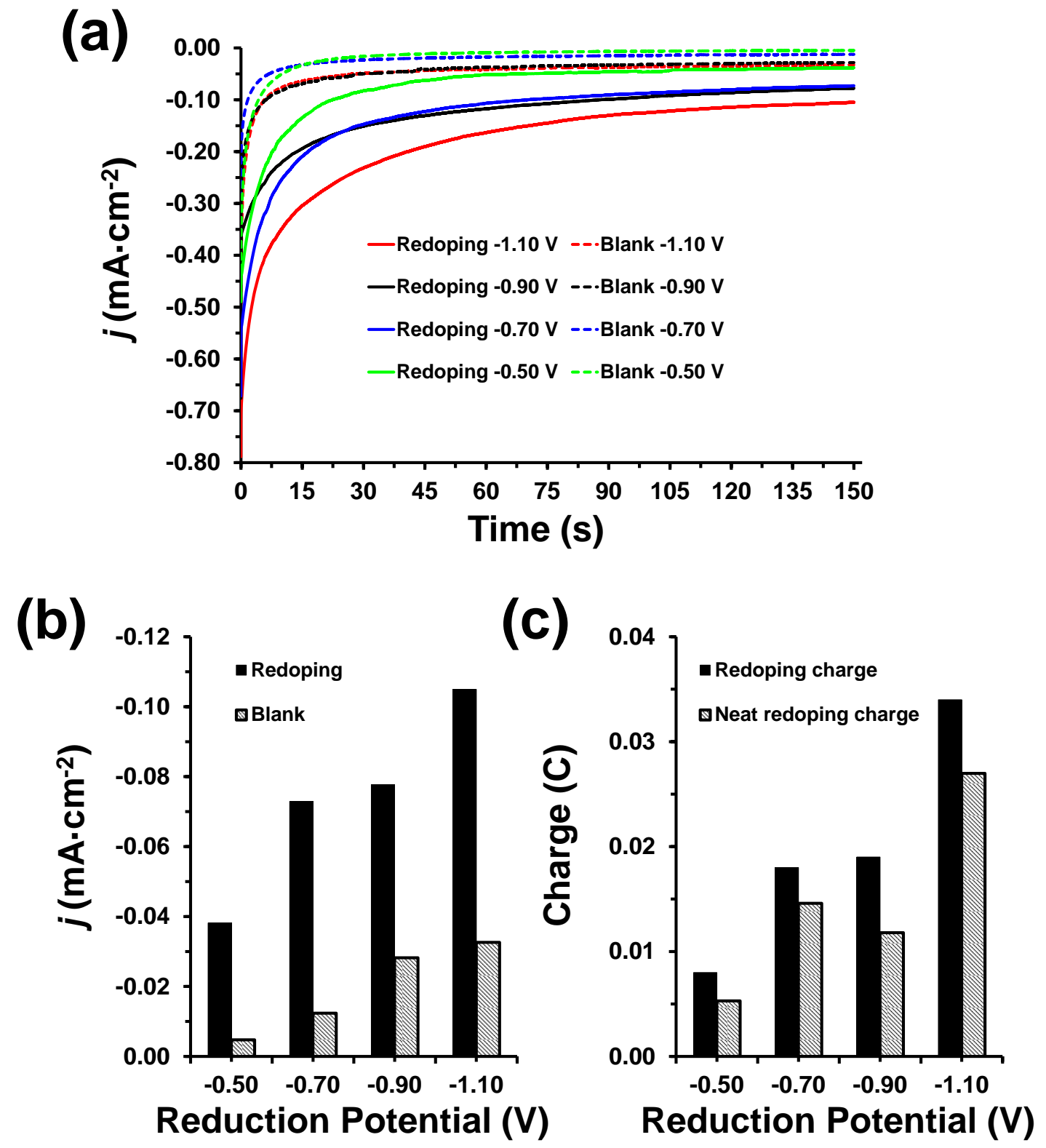

Figure 3 

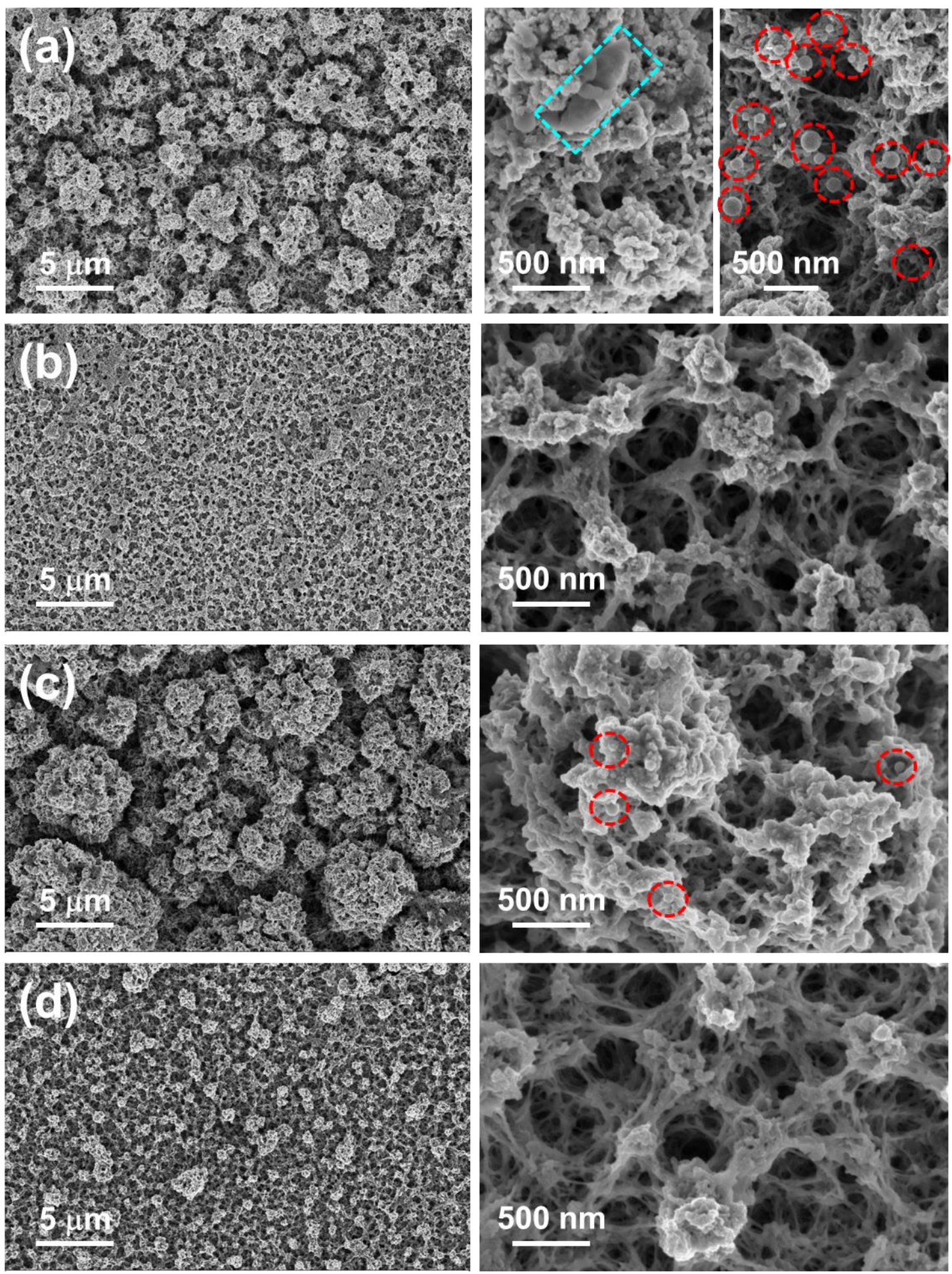

Figure 4 

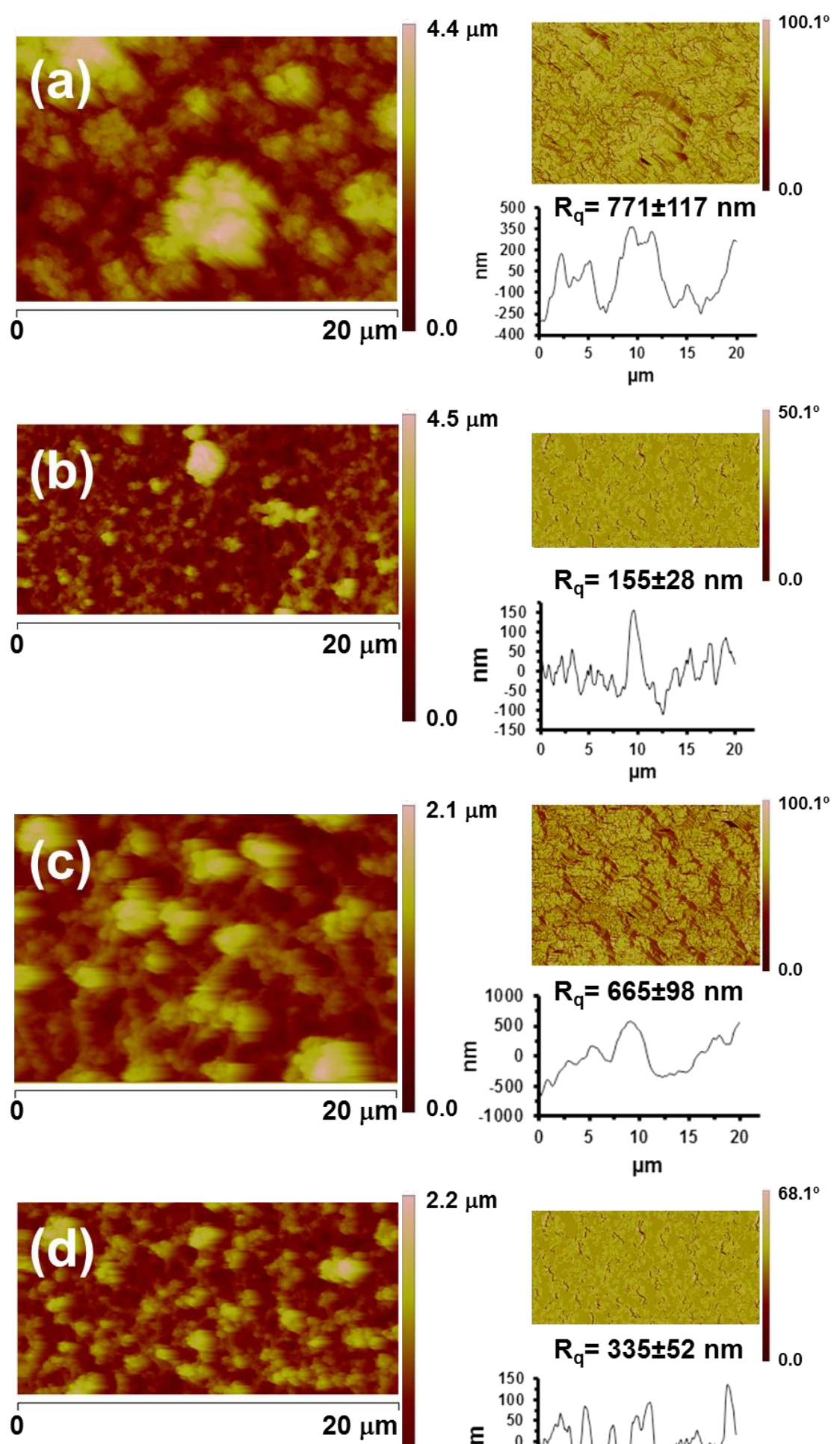

$2.2 \mu \mathrm{m}$
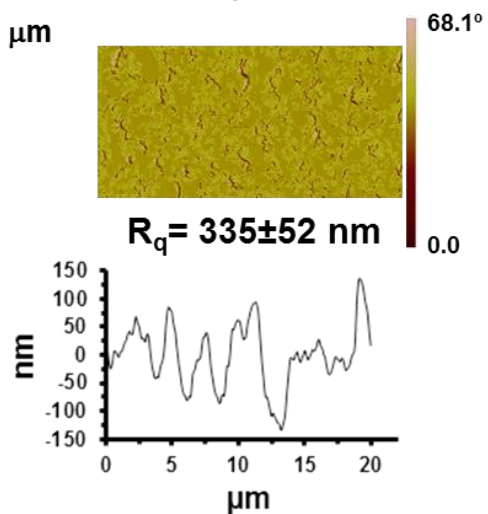

Figure 5 
(a)

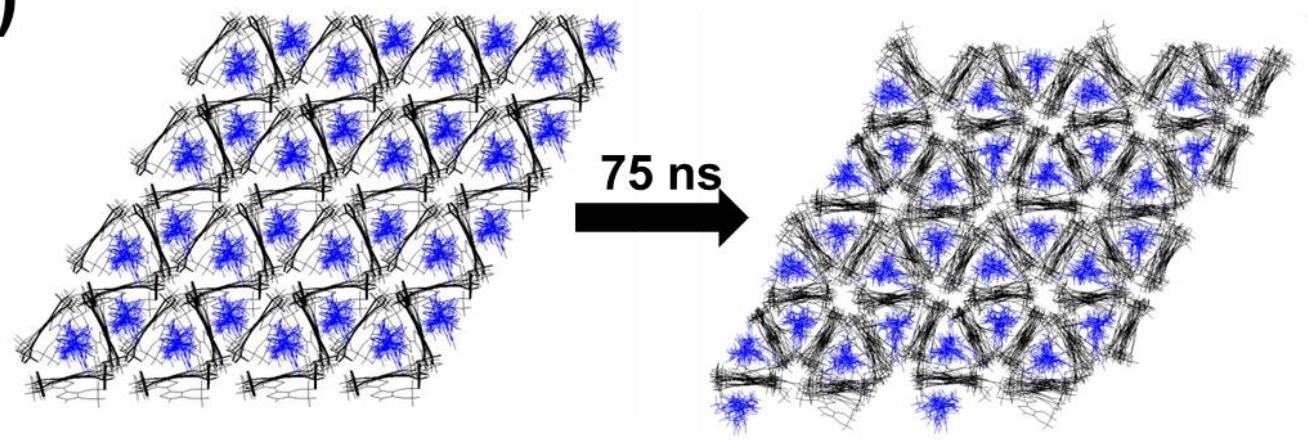

(b)

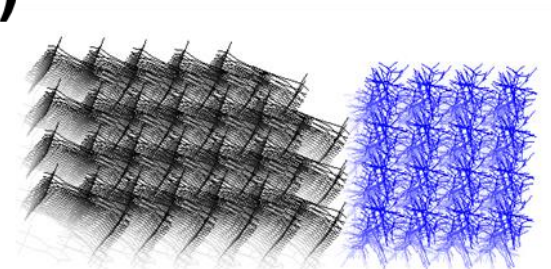

$75 \mathrm{~ns}$
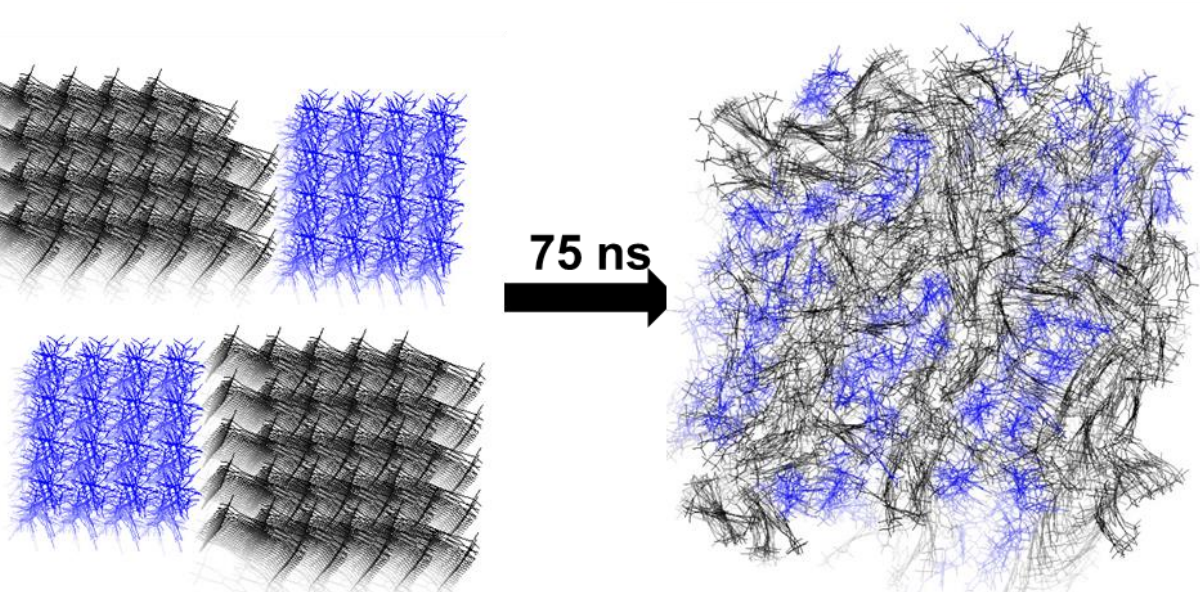

(c)

(d)
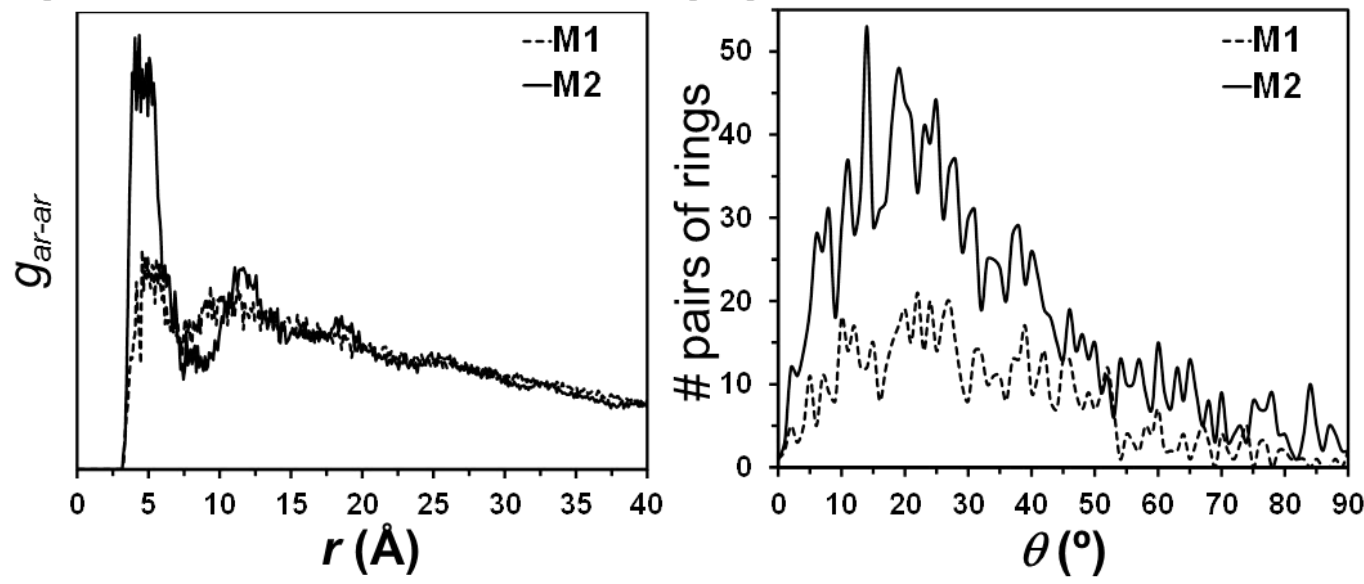

Figure 6 
(a)

(b)
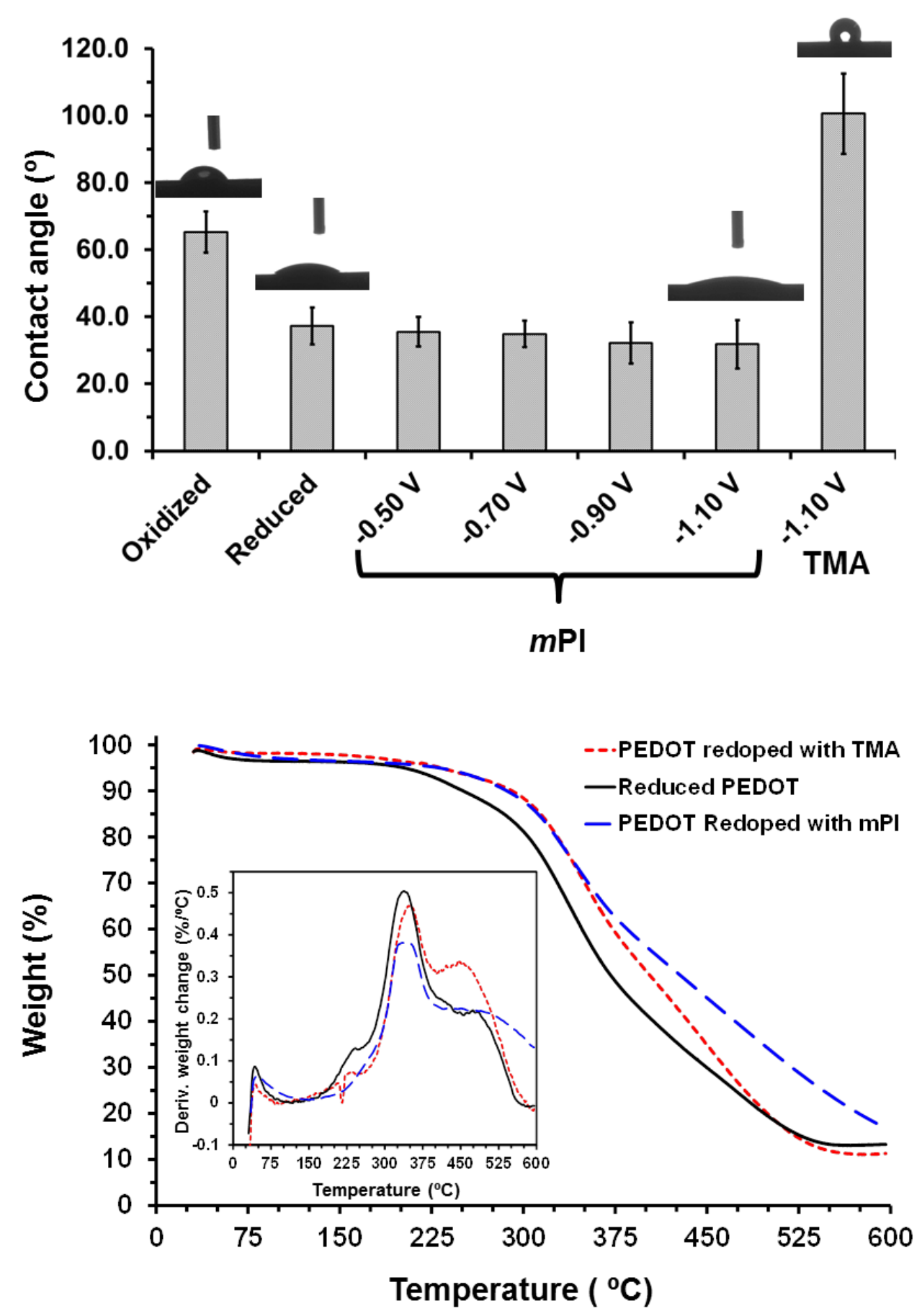

Figure 7 
Graphical Abstract

n-DOPED PEDOT ELECTRODES

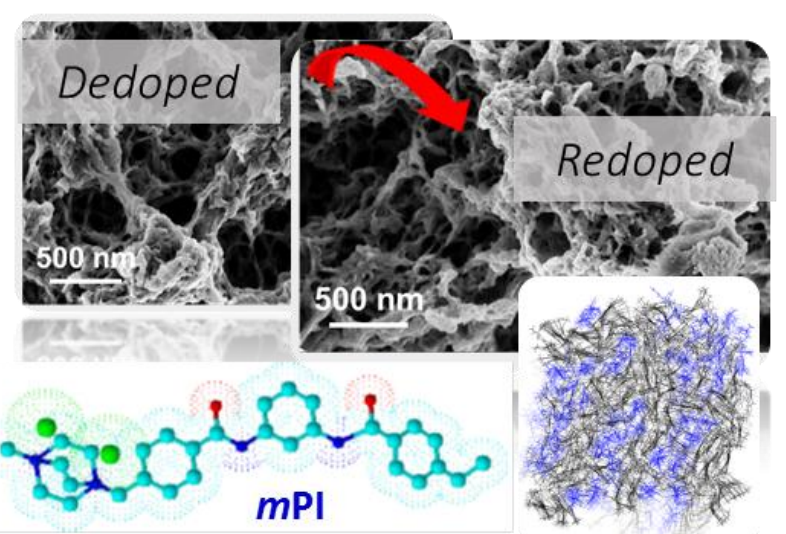

